\title{
HACIA UNA IMAGEN NO DEFORMADA DE LA ACTIVIDAD CIENTÍFICA
}

\author{
Daniel GIL PÉREZ, Isabel FERNANDEZ MONTORO \\ y Jaime CARRASCOSA ALIS \\ Universitat de Valencia
}

\section{Introducción}

Cabría suponer que quienes nos hemos formado en una facultad científica (Biología, Física, Química...) y ejercemos la enseñanza de las ciencias deberíamos haber adquirido - $-y$, por tanto, estaríamos en situación de transmitir - una imagen aceptablemente correcta de lo que supone hacer ciencia.

Numerosos estudios, sin embargo, han mostrado que ello no es así y que la enseñanza -incluida la universitaria - transmite, por ejemplo, visiones empiro-inductivistas de la ciencia que se alejan notoriamente de la forma como se construyen los conocimientos científicos (Cleminson, 1990; Matthews, 1991; Stinner, 1992; Hodson, 1993; Pomeroy, 1993; Désautels et al, 1993; Koulaidis y Ogborn, 1995; Thomaz et al, 1996).

No es objeto de este trabajo analizar las razones de esta incoherencia, que remite, en primer lugar, al hecho bien establecido de que la enseñanza cientifica -incluida, insistimos, la universitaria - se ha reducido básicamente a la presentación de conocimientos ya elaborados, sin dar ocasión a los estudiantes de asomarse a las actividades características de la actividad científica (Yager y Penick, 1983; Burbules y Linn, 1991; Matthews, 1991; Songer y Linn, 1991; Brickhouse, 1994; Solomon, Duveen y Scott, 1994; Hammer, 1995).

Ello hace que las concepciones de los estudiantes -incluidos los futuros docentes- no lleguen a diferir de lo que suele denominarse una imagen 
"folk», "naif» o "popular», socialmente aceptada, de la ciencia (Fernández, 2000), asociada a un supuesto "Método Cientifico", con mayúsculas, perfectamente definido.

Se podría argumentar que esta disonancia carece, en el fondo, de importancia, puesto que no ha impedido que los docentes desempeñemos nuestra tarea de transmisores de los conocimientos cientificos (que es la función social que nos ha sido encomendada). Sin embargo, las limitaciones de una educación científica centrada en la mera transmisión de conocimientos - limitaciones puestas de relieve por una abundante literatura, recogida en buena medida en los Handbooks ya aparecidos (Gabel, 1994; Fraser y Tobín, 1998; Perales y Cañal, 2000) - han impulsado investigaciones que han seńalado a las concepciones epistemológicas incorrectas como uno de los principales obstáculos para movimientos de renovación (Bell y Pearson, 1992; Furió, 1994; Cachapuz, 1995; Désautels y Larochelle, 1998a y b). Se ha comprendido así que «toda estrategia pedagógica adquiere sentido y relevancia en función, entre otros factores, de la opción epistemológica de su autor" (Désauteles et al., 1993). Ello ha convertido el estudio de dichas concepciones en una potente línea de investigación (Brickhouse, 1989 y 1990; Dushl y Wright 1989; Porlán, 1989; Cleminson, 1990; Burbules y Linn, 1991; Gallagher 1991; Loving 1991; Briscoe, 1991 y 1993; Brickhouse y Bodner, 1992; Gaskell, 1992; Linder, 1992; Désauteles et al., 1993; Carrascosa et al., 1993; Guilbert y Meloche, 1993; Hodson, 1993; Pomeroy, 1993; Ruba y Harkness, 1993; Ruggieri, Tarsitani y Vicentini, 1993; Acevedo, 1994; Lakin y Wellington, 1994; Abrams y Wandersee, 1995; Cachapuz, 1995; Fernández y Orozco, 1995; Hewson, Kerby y Cook, 1995; Koulaidis, y Ogborn, 1995; Hashweeh, 1996; Mellado, 1996, 1997 y 1998; Thomaz et al., 1996; Briscoe y Peters, 1997; Botton y Brown, 1998; Désauteles y Larochelle, 1998a; McComas, 1998a; Paixão y Cachapuz 1998 y 1999; Porlán y Rivero, 1998; Sutton, 1998; Lemberger, Hewson y Park, 1999).

Todo ello replantea, pues, la necesidad de establecer lo que puede entenderse como una visión mínimamente correcta de la actividad científica. Somos conscientes de la dificultad que entraña hablar de una "imagen correcta" de la ciencia, que parece sugerir la existencia de un supuesto método universal, de un modelo único de cambio científico (Estany, 1990). Es preciso, por supuesto, evitar cualquier interpretación de este tipo, pero ello no se consigue renunciando a hablar de las características de la actividad científica, sino con un esfuerzo consciente por evitar simplismos y deforma- 
ciones. Ello nos ha llevado a buscar, en primer lugar, un acuerdo básico en torno a aquello que debería evitarse, es decir, en torno a aquello que puede ser rechazado con claridad como contrario a lo que puede entenderse, en sentido amplio, como aproximación científica al tratamiento de problemas.

Se trata, en cierto modo, de aprehender por vía negativa — de rechazo de posibles deformaciones - una actividad compleja que parece difícil de caracterizar positivamente. Nuestra hipótesis ha sido que esta aproximación podría resultar más efectiva - al menos inicialmente- para establecer colectivamente las características esenciales de la actividad científica.

Abordaremos en un primer apartado esta búsqueda de visiones deformadas, susceptibles de concitar un amplio consenso en torno a lo que debe rechazarse cuando pretendemos adoptar actitudes científicas. Pero esta consideración de posibles deformaciones a evitar comporta implícitamente una caracterización positiva de la naturaleza de la ciencia que puede y debe hacerse explícita. Una caracterización que, por supuesto, no supone negar el amplio margen de ambiguiedad, no reducible a reglas fijas, propio de una actividad abierta y creativa, lo que constituye, sin duda, una primera característica a destacar.

Nuestra conjetura, además, es que dicha imagen se puede obtener directamente a partir de la consideración de lo que tienen en común los diversos planteamientos epistemológicos de autores como Popper (1962), Kuhn (1971), Bunge (1976), Toulmin (1977), Lakatos (1982), Laudan (1984), Giere (1988)... Hemos procedido con este fin a una búsqueda de dichos puntos comunes - dejando de lado los indudables matices y divergenciascon objeto de extraer algunas proposiciones básicas en torno a la actividad científica. Sin negar el interés de matices y divergencias, hemos puesto el acento en aquello en que existe consenso, como base de una visión de la ciencia que no caiga en notorios simplismos y deformaciones. Ello, pensamos, es suficiente - al menos en una primera aproximación- para orientar la actividad de quienes tenemos la responsabilidad, tanto de la alfabetización científica de los futuros ciudadanos y ciudadanas, como de la preparación inicial de los futuros científicos.

Dedicaremos el segundo apartado de este trabajo a la presentación de esta caracterización positiva de la actividad científica e intentaremos mostrar que dicha caracterización sale al paso de la totalidad de las deformaciones estudiadas en el primer apartado. Por último, nos referiremos a algunas implicaciones educativas de este esfuerzo de clarificación. 


\section{Visiones deformadas de la actividad científica}

Como ya hemos señalado, nuestro punto de partida para acercarnos a la naturaleza de la actividad científica - es decir, para comprender cómo se construyen y cambian los conocimientos científicos- ha sido el convencimiento de que, para ello, resulta útil comenzar reflexionando sobre las posibles deformaciones que la enseñanza de las ciencias podría estar transmitiendo, por acción o por omisión, acerca de dicha naturaleza de la ciencia. Una consideración explícita de dichas deformaciones puede ayudar, pensamos, a cuestionar concepciones y prácticas asumidas acríticamente - por impregnación ambiental- y a aproximarse a concepciones epistemológicas más correctas que, si son debidamente reforzadas, pueden incidir positivamente sobre la enseñanza.

Dos han sido, básicamente, las estrategias utilizadas para sacar a la luz las deformaciones en las que puede incurrir la imagen de la actividad científica proporcionada por la enseñanza de la ciencia. Por una parte, hemos procedido - en el contexto de talleres sobre la naturaleza de la ciencia y su papel en la enseñanza- a colocar a equipos de profesores en situación de investigadores que han de estudiar y analizar críticamente las concepciones docentes sobre la ciencia.

Una actividad básica en ese proceso ha consistido en solicitar a los equipos una enumeración - a título de primera conjetura, basada en una reflexión crítica sobre la práctica docente que les es familiar- de cuáles podrían ser las deformaciones en las que la enseñanza de las ciencias estaría incurriendo por acción u omisión. Podría pensarse que esta actividad ha de ser escasamente productiva ya que se está pidiendo a los responsables, en cierto modo, de las deformaciones, que investiguen cuáles pueden ser éstas. Sin embargo, nuestra hipótesis - y nuestra apuesta - ha sido que, al crearse una situación de investigación colectiva, los profesores podemos distanciarnos críticamente de nuestras concepciones y prácticas habituales, fruto de una impregnación ambiental que no habíamos tenido ocasión de analizar y valorar. Nuestra hipótesis, pues, es que esta reflexión colectiva de distintos equipos docentes va a proporcionar resultados coherentes y valiosos para conocer posibles deformaciones de la naturaleza de la ciencia a las que habría que prestar atención.

El resultado de este trabajo, que ha sido realizado con numerosos grupos de profesores en formación y en activo (pues forma parte de nuestras estrategias habituales de formación del profesorado de ciencias), es que las deforma- 
ciones conjeturadas son siempre las mismas; más aún, no sólo se señalan sistemáticamente las mismas deformaciones, sino que se observa una notable coincidencia en la frecuencia con que cada una es mencionada.

Una segunda estrategia utilizada para conocer estas posibles deformaciones de lo que es hacer ciencia ha consistido en analizar artículos sobre educación cientifica relacionados con la naturaleza de la ciencia y buscar en los mismos referencias a posibles errores y simplismos en la forma en que la enseñanza de la ciencia presenta dicha naturaleza. Hemos procedido, pues, a analizar varias docenas de artículos aparecidos entre 1984 y 1998 en revistas como Science Education, International Journal of Science Education, Journal of Research in Science Teaching, Enseñanza de las Ciencias, etc., así como trabajos recogidos en el International Handbook of Science Education, editado por Fraser y Tobin (1998) y en una recopilación de McComas (1998) con el título The nature of science in science education. Rationales and strategies.

Los resultados de este análisis bibliográfico son sorprendentemente coincidentes con las conjeturas de los equipos docentes en lo que se refieren a las deformaciones mencionadas y, en general, incluso a la frecuencia con que lo son (Fernández, 2000). Esta coincidencia básica refuerza nuestra hipótesis de la validez de una reflexión de los docentes debidamente apoyada. De hecho una multiplicidad de diseños experimentales (que incluyen desde la utilización de cuestionarios a entrevistas a profesores, pasando por el análisis de textos escolares y de diversos materiales elaborados por profesores en formación y en activo) nos ha permitido constatar que dichas deformaciones son transmitidas, efectivamente, por la educación científica (Fernández, 2000).

Podemos, pues, intentar aproximarnos a una imagen más correcta de la actividad científica, tomando en consideración estas deformaciones, es decir, tratando explícitamente de no incurrir en las mismas ni por acción ni por omisión. Expondremos brevemente, a continuación dichas deformaciones, que expresan, en conjunto, una imagen ingenua profundamente alejada de lo que supone la construcción de conocimientos científicos, pero que ha ido consolidándose hasta convertirse en un estereotipo socialmente aceptado que, insistimos, la propia educación científica refuerza por acción u omisión.

1. Quizás la deformación que ha sido estudiada en primer lugar, y la más ampliamente señalada en la literatura, es la que podríamos denominar una concepción empiro-inductivista y ateórica. Una concepción que resalta el papel de la observación y de la experimentación "neutras» (no contaminadas 
por ideas aprioristicas), e incluso del puro azar, olvidando el papel esencial de las hipótesis como focalizadoras de la investigación y de los cuerpos coherentes de conocimientos (teorías) disponibles, que orientan todo el proceso.

En efecto, numerosos estudios han mostrado las discrepancias entre la visión de la ciencia proporcionada por la epistemología contemporánea y ciertas concepciones docentes, ampliamente extendidas, marcadas por un empirismo extremo (Giordan, 1978; Hodson, 1985; Nussbaum, 1989; Cleminson, 1990; King, 1991; Stinner, 1992; Désauteles et al., 1993; Lakin y Wellington, 1994; Hewson, Kerby y Cook, 1995; Jiménez Aleixandre, 1995; Thomaz et al., 1996; Izquierdo, Sanmartí y Espinet 1999...).

Estas concepciones empiro-inductivistas de la ciencia afectan a los mismos cientificos - pues, como explica Mosterín (1990) sería ingenuo pensar que uson siempre explícitamente conscientes de los métodos que usan en su investigación»- así como, logicamente, a los mismos estudiantes (Gaskell, 1992; Pomeroy, 1993; Roth y Roychondhury, 1994; Solomon, Duveen y Scott 1994; Abrams y Wanderse, 1995; Traver, 1996; Roth y Lucas, 1997; Désautels y Larochelle, 1998b). Conviene señalar que esta idea, que atribuye la esencia de la actividad cientifica a la experimentación, coincide con la de "descubrimiento" científico, transmitida, p.e., por los cómics, el cine y, en general, por los medios de comunicación, prensa, revistas, televisión, (Lakin y Wellington, 1994). Dicho de otra manera, parece que la visión de los profesores $\longrightarrow$ la que proporcionan los libros de texto (Selley, 1989; Stinner, 1992) - no es muy diferente, en lo que respecta al papel atribuido a los experimentos, de lo que hemos denominado la imagen «ingenua» de la ciencia, socialmente difundida y aceptada.

Cabe señalar que aunque ésta es, sin duda, la deformación más estudiada y criticada en la literatura, tanto en la década de los años setenta como en el periodo 1984-1998 analizado (con más de 60 trabajos que hacen referencia a la misma ${ }^{1}$ ), son pocos los equipos docentes que hacen referencia a esta posi-

1 Trabajos en los que se critica la visión empiro-inductivista y ateóricade la ciencia.

NADEAU y DESAUTELS (1984); HODSON (1985) y (1992a); OTERO (1985); BRONOwSKI (1987); GioRdAN y DE VECCHI (1987); Gould (1987); SELLEY (1989); BRICKHOUSE (1989, 1990 y 1994); Jacoby y Spargo (1989); Cleminson (1990); Koballa, Crawley y Shrigley (1990); Burbules y LINN (1991); GallaGHER (1991); KING (1991); LOVING (1991); MATTHEWS (1991); SOlOMON (1991); GASKELl (1992); LEDERMAN (1992); LINDER (1992); Stinner (1992); Tobin, Tippins y Gallard, (1992); Carrascosa et al. (1993); Désautels 
ble deformación. Ello puede interpretarse como índice del peso que continúa teniendo esta concepción empiro-inductivista en el profesorado de ciencias. Es preciso tener en cuenta a este respecto que, pese a la importancia dada (verbalmente) a la observación y experimentación, en general la enseñanza es puramente libresca, sin apenas trabajo experimental. Ello favorece que la experimentación conserve para profesores y estudiantes el atractivo de una «revolución pendienten, como hemos podido percibir en entrevistas realizadas a profesores en activo (Fernández, 2000). De hecho los intentos de renovación de la educación científica iniciados en los ańos 60 incurrían, en sus mismas denominaciones — «aprendizaje por descubrimiento», «aprendizaje de los procesos cientificoss... - en esta visión ateórica, centrada en el supuesto Método Científico, con olvido de los contenidos (Gil 1983; Sanmartí et al 1990).

2. Una segunda deformación ampliamente recogida en la literatura (cerca de 40 artículos en el periodo analizado ${ }^{2}$ ) es la que transmite una visión rígida (algorítmica, exacta, infalible...). Se presenta el "Método Científico» como un conjunto de etapas a seguir mecánicamente. Se resalta, por otra parte, lo que supone tratamiento cuantitativo, control riguroso, etc., olvidando -0 , incluso, rechazando- todo lo que significa invención, creatividad, duda... Ello se pone particularmente en evidencia en lo que respecta a la evaluación: como afirma Hodson (1992b), la preocupación obsesiva por evitar la

ET AL (1993); Gil (1993 y 1994a); GUILBERT y Meloche (1993); HodSON (1993); POMEROY (1993); RUba y HaRKNERSS (1993); RUGgIERI, TARSITANI y VICENTINI (1993); ACEVEDO (1994); DUSCHL (1994); FURIO (1994); LAKIN y WELLINGTON (1994); ROTH y RoYchoudhury (1994); SOlomon, Duveen y SCOTT (1994); TOBIN, Tippins y HoOK, (1994); FERnANDEZ y OrozCo (1995); HEWSON, KERBY y COOK (1995); JiMENEZ (1995); KOULADIS Y OGBORN (1995); OROZCO Y FERNÁNDEZ (1995); GIL (1996); HASHWEEH (1996); TOMAZ eT al (1996); CAMPO y CACHAPUZ 1997; Boersema (1998); Cobern y LoVING (1998); DAWKINS y GLATHORN (1998); HAMMERICH (1998); LEDERMAN y ABD-EL-KhaLICK (1998); MATSON y PARSONS (1998); MCCOMAS (1998a y 1998b); MCCOMAS y OLSON (1998); MCCOMAS ET AL (1998); MEITCHTRY (1998); NotT y Wellington (1998); PAIXÃo y CACHAPUZ 1998; PORLAN y RIVERO, (1998); SPECTOR, STRONG y LA PORTA (1998); SUTTON (1998); IZQUIERDO, SANMARTI y ESPINET (1999).

2 Trabajos que mencionan la visión rígida (algorítmica, exacta, infalible,...).

Aikenhead (1984); Hodson (1985); Porlan (1989); Gallagher (1991); Gaskell (1992); HODSON (1992a) (1992b); LINDER (1992); TOBIN, TIPPINS y GAllard, (1992); Carrascosa et al. (1993); Gil (1993); GuilberT y Meloche (1993); Hodson (1993); ACEVEDO (1994); BRICKHOUSE (1994); FURIO (1994); GIL (1994a); LAKIN y WELLINGTON (1994); Pedrinaci (1994); Solomon, DuveEn y SCOTt (1994); Tobin, Tippins y Hook (1994); Fernández y Orozco (1995); Orozco y Fernández (1995); Gil (1996); 
ambigüedad y asegurar la fiabilidad de las evaluaciones, distorsiona la naturaleza misma del trabajo cientifico, esencialmente difuso, incierto, intuitivo... La evaluación debería tener en cuenta dicha ambigüedad, no intentar eliminarla.

Se trata de una concepción ampliamente difundida entre el profesorado de ciencias, como hemos podido constatar utilizando diversos diseños experimentales (Fernández 2000). Así, en las entrevistas que hemos mantenido con profesores, una mayoría se ha referido al "Método Científico" como una secuencia de etapas definidas, destacando el rigor del mismo y el carácter exacto de los resultados obtenidos. Resultados semejantes han sido obtenidos por otros investigadores ${ }^{2}$.

Cabe señalar que ésta sí es una deformación ampliamente criticada por los equipos docentes, hasta el punto de que algunos, al rechazar ésta visión rígida y dogmática de la ciencia, hacen suyo un relativismo extremo, tanto metodológico ("todo vale», no hay estrategias especificas en el trabajo científico), como conceptual (no hay una realidad objetiva que permita contrastar la validez de las construcciones científicas: la única base en la que se apoya el conocimiento es el consenso de la comunidad de investigadores en ese campo); un relativismo del que encontramos apoyos en la literatura (Feyerabend 1989) y, por supuesto, críticas (Izquierdo, Sanmartí y Espinet 1999).

3. Muy ligada a esa visión rígida, podemos mencionar la visión aproblemática y ahistórica (ergo dogmática y cerrada): Se transmiten conocimientos ya elaborados, sin mostrar cuáles fueron los problemas que generaron su construcción, cuál ha sido su evolución, las dificultades, etc., ni mucho menos aún, las limitaciones del conocimiento cientifico actual o las perspectivas abiertas. Se pierde así de vista que, como afirma Bachelard (1938), "todo conocimiento es la respuesta a una cuestión", a un problema, lo que dificulta captar la racionalidad del proceso cientifico.

Se trata de una concepción que la enseñanza de la ciencia refuerza por omisión. En efecto, los profesores de ciencias, tanto al ser entrevistados como cuan-

SHWEEH (1996); TOMAZ ET AL. (1996); BOERSEMA (1998); COBERN y LOVING (1998); DAWKINS y GLATHORN (1998); HAMMERICH (1998); LEDERMAN y ABD-EL-KHALICK (1998); Matson y Parsons (1998); MCCOMAS (1998a y1998b); MCCOMAS y OLSON (1998); Meitchtry (1998); NotT y Wellington (1998); Paixáo y CaChapuz 1997; Porlan y Rivero, (1998); Spector, Strong y La Porta (1998); Sutton (1998). 
do resuelven distintos tipos de cuestiones relativas a la forma de introducir los conocimientos científicos, no hacen referencia a los problemas que están en el origen de la construcción de dichos conocimientos. Es decir, la visión que transmiten, en general, incurre por omisión en una visión aproblemática; y lo mismo se aprecia en los libros de texto (Fernández 2000).

Más de 35 artículos se refieren a esta concepción ${ }^{3}$, que es también critica$\mathrm{da}$ frecuentemente por los equipos docentes, cuando se favorece su reflexión crítica, recordemos, proponiéndoles que participen en una investigación en torno a posibles deformaciones de la actividad cientifica transmitidas por la educación.

4. Una deformación que apenas es mencionada por los equipos docentes y que ha sido escasamente tratada por la investigación (menos de una docena de artículos hacen alguna referencia a la misma ${ }^{4}$ ) es la consistente en una visión exclusivamente analítica, que resalta la necesaria parcelación inicial de los estudios, su carácter acotado, simplificatorio, pero que olvida los esfuerzos posteriores de unificación y de construcción de cuerpos coherentes de conocimientos cada vez más amplios, o, el tratamiento de problemas "puente" entre distintos campos de conocimiento que pueden llegar a unirse, como ha ocurrido tantas veces.

Quizás esta escasa atención a una deformación que nos parece particularmente grave pueda ser debida a que las propuestas de tratamiento interdisci-

3 Trabajos que incluyen referencias relativas a la visión aproblemática y ahistórica (ergo dogmática y cerrada)

Otero (1985); Gagliardi y Giordan (1986); Giordan y De Vecchi (1987); Porlan (1989); Cleminson (1990); Koballa, Crawley y Shrigley (1990); Garcla Cruz (1991); Linder (1992); STINNER (1992); Tobin, Tippins y Gallard, (1992); Carrascosa et al. (1993); Gil (1993); GuILBERT y MELOCHE (1993); ACEVEDO (1994); BRICKHOUSE (1994); Furio (1994); Gil (1994A); LAKIN, y Wellington (1994); PEDRinaCi (1994); Roth y ROYCHOUDHURY (1994); SOLOMON, DUVEEN y SCOTT (1994); TOBIN, TIPPINS y HOOK (1994); ABRAMS y WANDERSEE (1995); FERNANDEZ y OROZCO (1995); OROZCO Y FERNANDEZ (1995); GIl (1996); Hashweeh (1996); TOMAZ ET al (1996); CaMPo y CaCHapuz 1997; Boersema (1998); DaWkINS y GLATHORN (1998); MATSON y PARSONS (1998); MCCOMAS (1998a y 1998b); MCCOMAS y Olson (1998); MeITCHTRY (1998); PaIXÃo y CaCHapuz 1998; PORLAN y RIVERO, (1998); SUUTTON (1998).

4 Trabajos en los que se presta atención a la visión exclusivamente analítica:

MatTHEWS (1991); HodSON (1992a); CARRASCOSA ET AL. (1993); Gil (1993); FuRIO (1994); Gil (1994A); FERNANDEZ y OROZCO (1995); OrOzCO y FERNANDEZ (1995); GIL (1996); MCCOMAS y Olson (1998); SPECTOR, StRONG y LA PORTA (1998). 
plinar e incluso de enseñanza integrada de las ciencias han sido ampliamente difundidas y parecen gozar de una buena aceptación (al menos verbal) entre los profesores. A nuestro entender, sin embargo, dichas propuestas incurren, a menudo, en un error de signo contrario al de la visión analítica, pero no menos grave, consistente en tomar la unidad de la materia como punto de partida, olvidándose que el establecimiento de dicha unidad constituye una conquista reciente y nada fácil de la ciencia (Gil et al, 1991; Gil, 1994b). Recordemos por ejemplo la fuerte oposición a las concepciones unitarias en Astronomía (heliocentrismo), Biología (evolucionismo) o en Química Orgánica (síntesis orgánica).

En cualquier caso, el olvido de los procesos de unificación como característica fundamental de la evolución de los conocimientos científicos constituye un auténtico obstáculo, en la educación cientifica habitual. En efecto, hemos podido constatar (Fernández, 2000) que más de un $80 \%$ de los profesores y de los libros de texto incurren, por omisión, en esta deformación, olvidando destacar, p.e., la unificación que supuso la síntesis newtoniana de las mecánicas celeste y terrestre, rechazada durante más de un siglo con condenas a la obra de Copérnico o Galileo.

5. Una deformación a la que tampoco hacen referencia, a menudo, los equipos docentes $-y$ que es la segunda menos mencionada en la literatura 5 tras la visión exclusivamente analítica - es la que transmite una visión acumulativa, de crecimiento lineal de los conocimientos científicos: el desarrollo científico aparece como fruto de un crecimiento lineal, puramente acumulativo (Izquierdo, Sanmartí y Espinet 1999), ignorando las crisis y las remodelaciones profundas, fruto de procesos complejos que no se dejan ahormar por ningún modelo definido de cambio científico (Giere, 1998; Estany, 1990).

5 Trabajos en los que se sale al paso de una visión meramente acumulativa, de crecimiento lineal, de los conocimientos científicos.

Porlan (1989); Cleminson (1990); Fillon (1991); Carrascosa et al. (1993); Gil (1993); GUILbERT y MeloChe (1993); RUgGIERI, TARSITANI y VICENTINI (1993); BRICKHOUSE (1994); Furio (1994); Gil (1994A); PedrinaCi (1994); Fernandez y OrozCo (1995); OROZCO Y FERNANDEZ(1995); GIL (1996); HASHWEEH (1996); TOMAZ ET AL., (1996); Boersema (1998); MATSON y PARSONS (1998); MCCOMAS (1998A y 1998B); MCCOMAS y OLSON (1998); MCCOMAS ET AL. (1998); MEITCHTRY (1998); NOTT y WELLINGTON (1998); Porlan y RIVERo, (1998); SPECTOR, STRONG y LA PORTA (1998); IzQuiERDO, SANMARTt y ESPINET (1999). 
Esta deformación es complementaria, en cierto modo, de lo que hemos denominado visión rígida, aunque deben ser diferenciadas: mientras la visión rígida o algorítmica se refiere a cómo se concibe la realización de una investigación dada, la visión acumulativa es una interpretación simplista de la evolución de los conocimientos científicos, a la que la enseñanza suele contribuir al presentar los conocimientos hoy aceptados sin mostrar cómo dichos conocimientos han sido alcanzados, ni referirse a las frecuentes confrontaciones entre teorías rivales, ni a los complejos procesos de cambio.

6. Una de las deformaciones más frecuentemente señaladas por los equipos docentes, y también más tratadas en la literatura ${ }^{6}$, es la que transmite una visión individualista y elitista de la ciencia. Los conocimientos científicos aparecen como obra de genios aislados, ignorándose el papel del trabajo colectivo, de los intercambios entre equipos... En particular se deja creer que los resultados obtenidos por un sólo científico o equipo, pueden bastar para verificar o falsar una hipótesis o, incluso, toda una teoría.

A menudo se insiste explícitamente en que el trabajo científico es un dominio reservado a minorías especialmente dotadas, transmitiendo expectativas negativas hacia la mayoría de los alumnos, con claras discriminaciones de naturaleza social y sexual (la ciencia es presentada como una actividad eminentemente "masculina»).

Se contribuye, además, a este elitismo escondiendo la significación de los conocimientos tras presentaciones exclusivamente operativistas. No se realiza un esfuerzo por hacer la ciencia accesible (comenzando con tratamientos cualitativos, significativos), ni por mostrar su carácter de construcción

6 Trabajos en los que se critica la visión individualista y elitista de la ciencia.

AIKenHeAd (1984); Gagliardi y GioRdaN (1986); PENICK y Yager (1986); Cleminson (1990); HODSON (1992A) y (1992B); NEWTON Y NEWTON (1992); STINNER (1992); CARRASCOSA ET AL. (1993); Gil (1993); GUILBERT y MELOCHE (1993); RUGGIERI, TARSITANI y VICENTINI (1993); BRICKHOUSE (1994); FURIO (1994); GIL (1994A); LAKIN y WELLINGTON (1994); TOBIN, TIPPINS y HOOK (1994); ABRAMS y WANDERSEE (1995); FERNANDEZ y OROZCO (1995); HEWSON, KERBY y COOK (1995); OROZCO y FERNÁNDEZ (1995); GIL (1996); TOMAZ ET AL. (1996); MATTEWS (1997); ROTH Y LUCAS (1997); BOERSEMA (1998); COBERN Y LOVING (1998); DAWKINS y GLATHORN (1998); HAMMERICH (1998); LEDERMAN y ABD-EL-KHALICK (1998); MATSON y PARSONS (1998); MCCOMAS (1998 a y b); MCCOMAS y OLSON (1998); MeITCHTRY (1998); NotT y Wellington (1998); PORLAN y RIVERO, (1998); SPECTOR, STRONG y LA PORTA (1998); SUTTON (1998). 
humana, en la que no faltan confusiones ni errores, como los de los propios alumnos.

En algunas ocasiones nos encontramos con una deformación de signo opuesto que contempla la actividad científica como algo sencillo, próximo al sentido común, olvidando que la construcción científica parte, precisamente, del cuestionamiento sistemático de lo obvio (Bachelard, 1938).

7. Por último nos referiremos a la deformación que transmite una visión descontextualizada, socialmente neutra de la ciencia ${ }^{7}$ : Se olvidan las complejas relaciones entre ciencia, tecnología y sociedad (CTS) y se proporciona una imagen de los científicos como seres "por encima del bien y del mal", encerrados en torres de marfil y ajenos a las necesarias tomas de decisión. Aunque en los últimos años los medios de comunicación se hacen frecuentemente eco de noticias acerca de, por ejemplo, los problemas del medio ambiente provocados por determinados desarrollos científicos no sometidos al "principio de prudencia", hemos podido constatar que un elevado porcentaje de profesores no toma en consideración esta dimensión de la actividad científica. Ello es particularmente notorio en lo que se refiere a las actividades utilizadas para evaluar el aprendizaje, lo que en definitiva muestra la escasa importancia que se le concede (Alonso, Gil y Martínez Torregrosa, 1992).

Sefialemos, no obstante, que los textos de la nueva Educación Secundaria Obligatoria comienzan a incluir mayor número de referencias a las implicaciones CTS de los desarrollos científicos.

7 Trabajos que se refieren a la visión descontextualizada, socialmente neutra de la actividad cientifica.

AIKENHEAD (1984); Gaglardi y Giordan (1986); BRUsh (1989); Cleminson (1990); Garcia CRUz (1991); GASKell (1992); HodSON (1992a) y (1992b); LiNDER (1992); CARRASCOSA ET AL. (1993); GIl (1993); GUILBERT y MELOCHE (1993); RUBA y HARKNERSS (1993); RUGGIERI, TARSITANI y VICENTINI (1993); ACEVEDO (1994); BRICKHOUSE (1994); FURIO (1994); Gil (1994A); ABRAMS y WANDERSEE (1995); FERNANDEZ y OROZCO (1995); OrOzCO y FerNANDEZ (1995); GIL (1996); TOMAZ ET AL. (1996); CAMPO y CACHAPUZ 1997; MATTEWS (1997); BoERSEMA (1998); COBERN y LOVING (1998); DaWKINS y GLATTHORN (1998); Lederman y ABD-EL-Khalick (1998); MATSON y PARSONS (1998); MCCOMAS (1998a y 1998b); MCCOMAS y OLSON (1998); MCCOMAS ET AL (1998); MEITCHTRY (1998); NOTT y Wellington (1998); Paixáo y CaChapuz 1998; Porlan y Rivero, (1998); SPECTOR, STrong y LA PORTA (1998); SutTon (1998); IzQUIERDO, SANMARTI y ESPINET (1999). 
Éstas son, en síntesis las siete grandes deformaciones que hemos visto tratadas en la literatura y que son mencionadas como fruto de la reflexión (auto)crítica de los equipos docentes. Son también las deformaciones que hemos visto reflejadas en la docencia habitual en un estudio detenido que ha utilizado cerca de 20 diseńos experimentales (Fernández, 2000).

Hemos de llamar la atención sobre el hecho de que estas deformaciones no constituyen una especie de "siete pecados capitales" distintos y autónomos; por el contrario, resulta lógico suponer que si existe una serie de visiones deformadas acerca de la ciencia, dichas visiones no constituirán concepciones absolutamente autónomas, sino que, al igual que se ha mostrado en el caso de las preconcepciones de los estudiantes en un determinado dominio (Driver y Oldham, 1986), formarán un esquema conceptual relativamente integrado. Parece razonable, p.e., que una visión individualista y elitista de la ciencia apoye implícitamente la idea empirista de "descubrimiento" $y$ contribuya, además, a una lectura descontextualizada, socialmente neutra, de la actividad cientifica (realizada por "genios" solitarios). Del mismo modo, por citar otro ejemplo, una visión rígida, algorítmica, exacta, de la ciencia pueden reforzar una interpretación acumulativa, lineal, del desarrollo cientifico, ignorando las crisis y las revoluciones cientificas.

Así pues, estas concepciones aparecen asociadas entre sí, como expresión de una imagen ingenua de la ciencia que se ha ido decantando, pasando a ser socialmente aceptada. De hecho esa imagen tópica de la ciencia parece haber sido asumida incluso por numerosos autores del campo de la educación, que critican como características de la ciencia lo que no son sino visiones deformadas de la misma. Asl, p.e., Kemmis y McTaggert (1982) (citado por Hodson, 1992a) critican a la «investigación convencional"(o «académican) su carácter "neutral", su preocupación exclusiva por "acumular conocimientos» (sin atención a ula mejora de la práctica"), su limitación a "un mero procedimiento de resolución de problemas" (olvidando el planteamiento de los mismos), etc., etc. Kemmis y McTaggert insisten reiteradamente en su crítica a la investigación académica, atribuyéndole deformaciones y reduccionismos que los autores dan por sentado que corresponden al "método cientifico" utilizado por "las ciencias naturales».

Incluso entre algunos investigadores en didáctica de la ciencia parece aceptarse que la ciencia clásica sería puramente analítica, "neutra", etc. Ya no se trata de que la enseñanza haya transmitido esas concepciones reduccionistas, 
empobrecedoras, sino que toda la ciencia clásica tendría esos defectos (García, 1995).

Pero, ¿̇cómo se puede afirmar que la ciencia clásica es -como suele afirmarse- puramente analítica, si su primer edificio teórico, significó la integración de dos universos considerados esencialmente distintos, derribando la supuesta barrera entre el mundo celeste y el sublunar? Una integración además, que implicaba desafiar dogmas, tomar partido por la libertad de pensamiento, correr riesgos de condenas, de inclusión en el Index Librorum Prohibitorum... y otros más graves. Y no es sólo la mecánica: toda la ciencia clásica puede interpretarse como la superación de supuestas barreras, la integración de dominios separados (por el sentido común y por los dogmas). Pensemos en la teoría de la evolución de las especies; en la síntesis orgánica (jen el siglo XIX todavía se sostenía la existencia de un "elan vital" y se negaba la posibilidad de sintetizar compuestos orgánicos!); en el electromagnetismo que mostró los vínculos entre electricidad, magnetismo y optica; en los principios de conservación y transformación de la masa y de la energía, aplicables a cualquier proceso. (Gil et al., 1991). ¿Dónde está el carácter puramente analítico? ¿Dónde está el carácter neutro, aséptico, de esa ciencia? Hay que reconocer que, al menos, no toda la ciencia clásica ha sido así. Parece más apropiado, pues, hablar de visiones (o, en todo caso, tendencias) deformadas de la ciencia, que atribuir esas características a toda la ciencia clásica.

Las concepciones docentes sobre la ciencia serían, pues, expresión de esa visión común, que los profesores de ciencias aceptaríamos implícitamente debido a la falta de reflexión crítica y a una educación cientifica que se limita, a menudo, a una simple transmisión de conocimientos ya elaborados. Ello no sólo deja en la sombra las características esenciales de la actividad científica, sino que contribuye a reforzar algunas deformaciones, como el supuesto carácter "exacto" (ergo dogmático) de la ciencia, o la visión aproblemática, etc. De este modo, la imagen de la ciencia que poseen los docentes se diferenciaría poco de las que puede expresar cualquier ciudadano y resulta muy alejada de las concepciones actuales acerca de la naturaleza de la ciencia. Pero, ¿̨uáles son esas "concepciones actuales" de la naturaleza de la ciencia?

Nuestra hipótesis, que ya hemos hecho explícita en la introducción a este trabajo, es que la lectura de los textos de diferentes epistemólogos contemporáneos va a mostrar - al margen de diferencias terminológicas, matices y divergencias en aspectos concretos, etc.- un consenso básico en una serie de 
elementos clave que dibujan una imagen de la ciencia radicalmente opuesta a las deformaciones estudiadas. Resumiremos dicho consenso en el siguiente apartado.

\section{Características esenciales de la actividad científica}

Ya hemos hecho referencia a que la naturaleza de la actividad cientifica ha dado lugar a serios debates, en los que se manifiestan notorias discrepancias entre los estudiosos (Popper, 1962; Kuhn, 1971; Bunge, 1976; Toulmin, 1977; Lakatos, 1982; Laudan, 1984, Feyerabend, 1989...). Ello genera, en ocasiones, una cierta perplejidad entre los profesores e investigadores en didáctica y lleva a plantear si tiene sentido hablar de una concepción correcta de la ciencia e incluso si merece la pena incluir la filosofía de la ciencia en los programas de formación de profesores (Martín, Kass y Brower, 1990; Stinner, 1992; Alters, 1997). Existen, sin embargo, algunos aspectos esenciales en los que se da una amplio consenso y que conviene destacar, evitando que los matices y discrepancias oculten lo que hay de común en los distintos planteamientos. Se trata, en definitiva, de evitar que algunos árboles nos impidan ver el bosque. Podemos resumir así los puntos de consenso:

1. En primer lugar hemos de referirnos al rechazo de la idea misma de "Método Científico", con mayúsculas, como conjunto de reglas perfectamente definidas a aplicar mecánicamente e independientes del dominio investigado. Con palabras de Bunge (1980): «La expresión (Método Científico) es engañosa, pues puede inducir a creer que consiste en un conjunto de recetas exhaustivas e infalibles...".

2. En segundo lugar hay que resaltar el rechazo generalizado de lo que Piaget (1970) denomina «el mito del origen sensorial de los conocimientos científicos», es decir, el rechazo de un empirismo que concibe los conocimientos como resultado de la inferencia inductiva a partir de "datos puros". Esos datos no tienen sentido en sí mismos, sino que requieren ser interpretados de acuerdo con un sistema teórico. Así, p.e., cuando se utiliza un amperímetro no se observa la intensidad de una corriente, sino la simple desviación de una aguja (Bunge, 1980). Se insiste, por ello, en que toda investigación y la misma búsqueda de datos vienen marcadas por paradigmas teóricos es decir, por visiones coherentes, articuladas que orientan dicha investigación. 
Es preciso insistir en la importancia de los paradigmas conceptuales, de las teorías, como origen y término del trabajo científico (Bunge, 1976), en un proceso complejo, no reducible a un modelo definido de cambio científico (Estany, 1990), que incluye eventuales rupturas, cambios revolucionarios (Kuhn, 1971), del paradigma vigente en un determinado dominio y surgimiento de nuevos paradigmas teóricos. $\mathrm{Y}$ es preciso también insistir en que los problemas científicos constituyen inicialmente "situaciones problemáticas" confusas: el problema no viene dado, siendo necesario formularlo de manera precisa, modelizando la situación, haciendo determinadas opciones de cara a simplificarlo más o menos para poder abordarlo, clarificando el objetivo, etc. $\mathrm{Y}$ todo esto partiendo del corpus de conocimientos que se posee en el campo específico en que se desarrolla el programa de investigación (Lakatos, 1989).

3. En tercer lugar hay que resaltar el papel jugado en la investigación por el pensamiento divergente, que se concreta en aspectos fundamentales y erróneamente relegados en los planteamientos empiro-inductivistas como son la invención de hipótesis y modelos, o el propio diseño de experimentos. No se razona, pues, en términos de certezas, más o menos basadas en "evidencias", sino en términos de hipótesis, que se apoyan, es cierto, en los conocimientos adquiridos, pero que son contempladas como simples «tentativas de respuesta» que han de ser puestas a prueba lo más rigurosamente posible, lo que da lugar a un proceso complejo, en el que no existen principios normativos, de aplicación universal, para la aceptación o rechazo de hipótesis o, más en general, para explicar los cambios en los conocimientos científicos (Giere 1998). Y si bien la obtención de evidencia experimental en condiciones definidas y controladas ocupa un lugar central en la investigación científica, es preciso relativizar dicho papel, que sólo cobra sentido, insistimos, con relación a las hipótesis a contrastar y a los diseños concebidos a tal efecto. En palabras de Hempel (1976), "al conocimiento científico no se llega aplicando un procedimiento inductivo de inferencia a datos recogidos con anterioridad, sino más bien mediante el llamado método de las hipótesis a título de intentos de respuesta a un problema en estudio y sometiendo luego éstas a la contrastación empírica». Son las hipótesis, pues, las que orientan la búsqueda de datos. Unas hipótesis que, a su vez, nos remiten al paradigma conceptual de partida, poniendo de nuevo en evidencia el error de los planteamientos empiristas.

4. Otro punto fundamental es la búsqueda de coherencia global (Chalmers, 1992). El hecho de trabajar en términos de hipótesis introduce exigencias suplementarias de rigor: es preciso dudar sistemáticamente de los resul- 
tados obtenidos y de todo el proceso seguido para obtenerlos, lo que conduce a revisiones continuas, a intentar obtener esos resultados por caminos diversos $y$, muy en particular, a mostrar su coherencia con los resultados obtenidos en otras situaciones. Es necesario llamar aquí la atención contra las interpretaciones simplistas de los resultados de los experimentos y contra un posible "reduccionismo experimentalista»: no basta con un tratamiento experimental para falsar o verificar - ni siquiera provisionalmente- una hipótesis; se trata sobre todo de la existencia, o no, de coherencia global con el corpus vigente de conocimientos.

De hecho uno de los fines más importantes de la ciencia estriba en la vinculación de dominios aparentemente inconexos. En efecto, en un mundo en el que lo primero que se percibe es la existencia de una gran diversidad de materiales y de seres, sometidos a continuos cambios, la ciencia busca establecer leyes y teorías generales que sean aplicables al estudio del mayor número posible de fenómenos. La teoría atómico molecular de la materia, la síntesis electromagnética, los principios de conservación y transformación, los esfuerzos que se realizan para unificar los distintos tipos de interacción existentes en la naturaleza, etc., son buenos ejemplos de esa búsqueda de coherencia y globalidad, aunque ello se deba realizar partiendo de problemas y situaciones particulares inicialmente muy concretas. El desarrollo científico, pues, entrana la finalidad de establecer generalizaciones aplicables a la naturaleza. Precisamente esa exigencia de aplicabilidad, de funcionamiento correcto para describir fenómenos, realizar predicciones, abordar y plantear nuevos problemas, etc., es lo que da creciente validez (que no certeza o carácter de verdad indiscutible) a los conceptos, leyes y teorías que se elaboran.

5. Por último, es preciso comprender el carácter social del desarrollo científico, lo que se evidencia no sólo en el hecho de que el punto de partida el paradigma teórico vigente es la cristalización de las aportaciones de generaciones de investigadores, sino también en que la investigación responde cada vez más a estructuras institucionalizadas (Bernal, 1967; Kuhn, 1971; Sutton, 1998; Matthews, 1991, 1994 y 1998) en las que la labor de los individuos es orientada por las líneas de investigación establecidas, por el trabajo del equipo del que forman parte, careciendo prácticamente de sentido la idea de investigación completamente autónoma. Más aún, el trabajo de los hombres y mujeres de ciencias - como cualquier otra actividad humana - no tiene lugar al margen de la sociedad en que viven y se ve afectado, lógicamente, por los problemas y circunstancias del momento histórico... sin que ello suponga caer en 
un relativismo extremo que resulta incapaz de explicar los éxitos del desarrollo cientifico-tecnológico (Giere, 1988). Del mismo modo la acción de los científicos tiene una clara influencia sobre el medio físico y social en que se inserta. Señalar esto puede parecer superfluo; sin embargo, la idea de que hacer ciencia es poco menos que una tarea de "genios solitarios" que se encierran en una torre de marfil, desconectando de la realidad, constituye una imagen tópica muy extendida y que la enseñanza, lamentablemente, no ayuda a superar, dado que se limita a la transmisión de contenidos conceptuales y, a lo sumo, entrenamiento en alguna destreza, pero dejando de lado los aspectos históricos, sociales... que enmarcan el desarrollo científico.

Se dibuja así una imagen imprecisa, nebulosa, de la metodología cientifica lejos de toda idea de algoritmo en la que nada garantiza que se llegará a un buen resultado, pero que representa, sin duda, la mejor forma de orientar el tratamiento de un problema científico (como atestiguan los impresionantes edificios teóricos construidos).

Puede decirse, en síntesis, que la esencia de la orientación científica - dejando de lado toda idea de "método" - se encuentra en el cambio de un pensamiento y acción basados en las "evidencias" del sentido común, a un razonamiento en términos de hipótesis, a la vez más creativo (es necesario ir más allá de lo que parece evidente e imaginar nuevas posibilidades) y más riguroso (es necesario fundamentar y después someter a prueba, cuidadosamente, las hipótesis, dudar de los resultados y buscar la coherencia global).

Es preciso tener presente, por otra parte, que una característica esencial de una aproximación científica es la voluntad explícita de simplificación y de control riguroso en condiciones preestablecidas, lo que introduce elementos de artificialidad indudables, que no deben ser ignorados ni ocultados: los científicos deciden abordar problemas resolubles y comienzan, para ello, ignorando consciente y voluntariamente muchas de las características de las situaciones estudiadas, lo que evidentemente les «aleja» de la realidad; y continúan alejándose mediante lo que, sin duda, hay que considerar la esencia del trabajo científico: la invención de hipótesis, la construcción de modelos imaginarios. El trabajo científico exige, pues, tratamientos analíticos, simplificatorios, artificiales. Pero ello no supone, como a veces se crítica, incurrir necesariamente en visiones parcializadas y simplistas: en la medida en que se trata de análisis y simplificaciones conscientes, se tiene presente la necesidad de síntesis y de estudios de complejidad creciente. Pensemos, por ejemplo, que el establecimiento de la 
unidad de la materia - que constituye un claro apoyo a una visión global, no parcializada - es una de las conquistas mayores del desarrollo científico de los últimos siglos: los principios de conservación y transformación de la materia y de la energía fueron establecidos, respectivamente, en los siglos XVIII y XIX, y fue sólo a fines del XIX que se produjo la fusión de tres dominios aparentemente autónomos —electricidad, óptica y magnetismo- en la teoría electromagnética, abriendo un enorme campo de aplicaciones que sigue revolucionando nuestra vida de cada día. Y no hay que olvidar que estos procesos de unificación han exigido, a menudo, actitudes críticas nada cómodas, que han tenido que vencer fuertes resistencias ideológicas e incluso persecuciones y condenas, como en los casos, bien conocidos, del heliocentrismo o del evolucionismo. La historia del pensamiento científico es una constante confirmación de que ésta es la forma correcta de hacer ciencia, profundizando en el conocimiento de la realidad en campos definidos, acotados; es esta profundización la que permite llegar a establecer lazos entre campos aparentemente desligados.

La idea de "método científico", en resumen, ha perdido hoy sus mayúsculas, es decir, su supuesta naturaleza de camino preciso - conjunto de operaciones ordenadas - e infalible, así como su supuesta neutralidad. Ello no supone, sin embargo, negar lo que de específico ha aportado la ciencia moderna al tratamiento de los problemas: la ruptura con un pensamiento basado en estudios puntuales, en las "evidencias" del sentido común y en seguridades dogmáticas, introduciendo un razonamiento que se apoya en un sistemático cuestionamiento de lo obvio y en una exigencia de coherencia global que se ha mostrado de una extraordinaria fecundidad.

Es fácil constatar, por otra parte, que la imagen de la ciencia que estos puntos de consenso dibujan, sale al paso del conjunto de los simplismos y deformaciones que hemos analizado en el apartado anterior. De hecho, cuando se presenta a los equipos docentes un resumen como el que acabamos de hacer, señalan de manera precisa cómo dicho texto sale al paso de cada una de las deformaciones. Ello refuerza, sin duda alguna, la validez de ambas aproximaciones y contribuye a afianzar el esfuerzo de clarificación realizado por los equipos docentes, que hemos descrito con detalle en otros trabajos (Fernández, 2000; Gil, Fernández y Carrascosa, 2000). Pero ¿qué interés puede tener este esfuerzo de clarificación?, ¿cuáles son sus implicaciones para una mejor educación científica? Abordaremos someramente — por razones de espacio- esta cuestión esencial en un último apartado. 
3. Algunas implicaciones para la enseñanza de las ciencias

Lograr una mejor comprensión de la actividad cientifica tiene, en sf mismo, un indudable interés, en particular para quienes somos responsables, en buena medida, de la educación científica de futuros ciudadanos de un mundo impregnado de ciencia y tecnología. Conviene recordar, sin embargo, que, como señalan Guilbert y Meloche (1993), "Una mejor comprensión por los docentes de los modos de construcción del conocimiento científico (...) no es únicamente un debate teórico, sino eminentemente prácticom. Se trata, pues, de facilitar que los equipos docentes comprendan la importancia práctica, para la docencia, del trabajo realizado y puedan sacar un mayor provecho del mismo. Hemos recurrido para ello a plantear a los equipos actividades como la siguiente, que presentamos a título de ejemplo:

"Tras la clarificación de la naturaleza del trabajo cientifico a que hemos procedido, estamos en situación de preguntarnos qué es lo que queremos potenciar en el trabajo de nuestros alumnos y alumnas y también de analizar si en nuestros materiales didacticos (o en los de otros colegas) se ignora alguin aspecto básico y se transmite de ese modo una vision deformada de la ciencia. Se trata, en definitiva de elaborar una red o 'parrilla' para orientar el diseño de actividades (o para facilitar su analisis), cuyos items recojan todos aquellos aspectos que consideremos conveniente contemplar para no caer en visiones simplistas de la ciencia».

Cabe esperar que, tras el trabajo de clarificación realizado, los equipos docentes hagan propuestas que se alejen de los habituales reduccionismos e incluyan aspectos que diversas líneas de investigación han señalado como fundamentales para favorecer un aprendizaje significativo de las ciencias, es decir, para favorecer la construcción de conocimientos científicos. Esperamos, más concretamente, que hagan alguna referencia a los aspectos recogidos en el cuadro 1 (Gil 1993). Los resultados que hemos obtenido en todas las ocasiones en que hemos planteado esta actividad, muestran que los distintos aspectos recogidos en dicho cuadro son, efectivamente, contemplados por los participantes en estos talleres de investigación dirigida.

Resultados similares se obtienen si, en vez de pedir "qué es lo que queremos potenciar en el trabajo de nuestros alumnos y alumnas", se solicita una reflexión centrada en la evaluación (Gil y Martínez Torregrosa, 1999). Naturalmente, los aspectos recogidos ahora apenas difieren de los considerados al centrar la atención en el currículo, puesto que al evaluar se muestra 
aquello a lo que se concede realmente importancia. Mediante actividades como éstas, los profesores hacen suya la necesidad de incluir en sus materiales, en las actividades que proponen para el trabajo en el aula, o en los ejercicios de evaluación, aspectos que enriquecen el proceso de enseñanza/aprendizaje de las ciencias y rompen con los habituales reduccionismos.

Cuadro 1. Aspectos a incluir en un currículo de ciencias para favorecer la construcción de conocimientos científicos

1. ¿Se presentan situaciones problemáticas abiertas (con objeto de que los alumnos puedan tomar decisiones para precisarlas) de un nivel de dificultad adecuado (correspondiente a su zona de desarrollo potencial)?

2 ¿Se plantea una reflexión sobre el posible interés de las situaciones propuestas que dé sentido a su estudio (considerando su relación con el programa general de trabajo adoptado, las posibles implicaciones CTS, etc.)?

¿Se presta atención, en general, a potenciar las actitudes positivas y a que el trabajo se realice en un clima próximo a lo que es una investigación colectiva (situación en la que las opiniones, intereses, etc. de cada individuo cuentan) y no en un clima de sometimiento a tareas impuestas por un profesor/»capataz»?

¿Se procura evitar toda discriminación (por razones étnicas, sociales...) y, en particular, el uso de un lenguaje sexista, transmisor de expectativas negativas hacia las mujeres?

3. ¿Se plantea un análisis cualitativo, significativo, que ayude a comprender y a acotar las situaciones planteadas (a la luz de los conocimientos disponibles, del interés del problema, etc) y a formular preguntas operativas sobre lo que se busca?

¿Se muestra, por otra parte, el papel esencial de las matemáticas como instrumento de investigación, que interviene desde la formulación misma de problemas al análisis de los resultados, sin caer en operativismos ciegos?

4 ¿ Se plantea la emisión de hipótesis, fundamentadas en los conocimientos disponibles, susceptibles de orientar el tratamiento de las situaciones y de hacer explícitas, funcionalmente, las preconcepciones? 
¿Se presta atención, en ese sentido, a la actualización de los conocimientos que constituyan prerrequisitos para el estudio emprendido?

¿Se propone, al menos, el manejo de alguna hipótesis?

¿Se presta atención a las preconcepciones (contempladas como hipótesis)?

5. ¿Se plantea la elaboración de estrategias (en plural), incluyendo, en su caso, diseños experimentales?

¿Se pide, al menos, la evaluación crítica de algún diseño, etc.? das...)?

¿Se presta atención a la actividad práctica en sí misma (montajes, medi-

¿Se potencia la incorporación de la tecnología actual a los diseños experimentales (ordenadores, electrónica, automación...) con objeto de favorecer una visión más correcta de la actividad científico-técnica contemporánea?

6 ¿Se plantea el análisis detenido de los resultados (su interpretación física, fiabilidad, etc), a la luz del cuerpo de conocimientos disponible, de las hipótesis manejadas y/o de los resultados de otros autores?

¿Se plantea alguna reflexión sobre los posibles conflictos entre algunos resultados $\mathrm{y}$ las concepciones iniciales?

¿Se favorece la "autorregulación" del trabajo de los alumnos?

¿Se promueve que los estudiantes cotejen su evolución conceptual y metodológica con la experimentada históricamente por la comunidad cientifica?

7. ¿Se plantea la consideración de posibles perspectivas (replanteamiento del estudio a otro nivel de complejidad, problemas derivados...)?

Se consideran, en particular, las implicaciones CTS del estudio realizado (posibles aplicaciones, repercusiones negativas...)?

¿Se pide la elaboración de "productos" (carteles, colecciones de objetos...)?

8 ¿ Se pide un esfuerzo de integración que considere la contribución del estudio realizado a la construcción de un cuerpo coherente de conocimientos, las posibles implicaciones en otros campos de conocimientos, etc.? 
¿Se pide algún trabajo de construcción de síntesis, mapas conceptuales, etc., que ponga en relación conocimientos diversos?

9. ¿Se presta atención a la comunicación como aspecto esencial de la actividad científica?

¿Se plantea la elaboración de memorias científicas del trabajo realizado?

¿Se pide la lectura y comentario crítico de textos científicos?

¿Se presta atención a la verbalización, solicitando comentarios significativos que eviten el "operativismo mudo"?

10. ¿Se potencia la dimensión colectiva del trabajo científico organizando equipos de trabajo y facilitando la interacción entre los equipos y la comunidad científica (representada en la clase por el resto de los equipos, el cuerpo de conocimientos ya construido, los textos, el profesor como experto...)?

¿Se hace ver, en particular, que los resultados de una sola persona o de un solo equipo no pueden bastar para verificar o falsar una hipótesis?

¿Se muestra el cuerpo de conocimientos disponible como la cristalización del trabajo realizado por la comunidad científica y la expresión del consenso alcanzado?

La consideración, realizada por los equipos docentes, de la conveniencia de introducir en la enseñanza de las ciencias aspectos como los contemplados en el cuadro 1 es un ejemplo de la incidencia positiva que puede tener la clarificación de la naturaleza de la ciencia. No pretendemos decir, por supuesto, que ello baste para orientar correctamente el proceso de enseñanza/aprendizaje de las ciencias, pero sí pensamos que constituye una valiosa contribución - un requisito sine qua non (Hodson, 1993, Tobin, Tippins y Hook, 1994; Eichinger, Abell y Dagher, 1997, Lyons, Freitag y Hewson, 1997; Desautels y Larochelle, 1998a y b) - que facilita, por otra parte, la asunción de las propuestas derivadas de otras líneas de la investigación didáctica, como las relativas a la forma de enfocar las prácticas de laboratorio, la resolución de problemas de lápiz y papel o la evaluación (Gil, Furió et al 1999). 


\section{Referencias bibliográficas}

ABRAMS, E. Y WaNDERSEE, J. H., (1995): How to infuse actual scientific researchpractices into science classroom instruction. International Journal of Science Education, Vol. 1, N. ${ }^{\circ} 6,683-694$.

ACEVEDO, J. A., (1994): Los futuros profesores de enseñanza secundaria ante la sociología y la epistemología de las ciencias: Un enfoque C/T/S. Revista Interuniversitaria de Formación del Profesorado, 19, 111-125.

AiKeNHEAD, G. S., (1984): Teacher decision making: The case of Prairie High. Journal of Research in Science Education, 21, 167-186.

Alonso M., Gil D. Y MARTINeZ TORREGROSA J., (1992): Concepciones espontáneas de los profesores de ciencias sobre la evaluación: obstáculos a superar y propuestas de replanteamiento, Revista de Enseñanza de la Física, 5, (2), 18-38.

ALTERS, B. J., (1997): Whose nature of science? Journal of Research in Science Teaching, $34,(1), 39-55$.

BACHELARD, G. , (1938): La Formation de L'esprit scientifique. (Vrin: Paris).

Bell, B. F, y PeARSON, J., (1992): Better Learning. International Journal of Science Education, 14 (3), 349-361.

Bernal, J. D., (1967): Historia social de la ciencia (Península: Barcelona).

BOERSEMA, D., (1998): The use of real and imaginary cases in comunicating the nature of science course outline. In W.F. McComas (Ed) The nature of science in science education. Rationales and estrategies. (Kluwer Academic Publishers. Netherland).

BOTTON, C. Y BROWN, C., (1998): The reliability of some VOSTS items when used with preservice secondary science teachers in England. Journal of Research in Science Teaching, 35, (1), 53-71.

BRICKHOUSE, N. W., (1989): The teaching of the philosophy of science in secondary lassrooms: Case studies of teachers' personal theories. International Journal of Science Education, (11), 437-449. BRICKHOUSE, N.W., 1990, Teachers' beliefs about the nature of science and their relationship to classroom practice. Journal of Teacher Education, 41, (3), 53-62.

BRICKHOUSE, N. W., (1994): Childrens observations, ideas and the developement of classroom. Theories about light. Journal of Research in Science Teaching, 31, (6), 639-656.

BRICKHOUSE, N. W. y BODNER, G. M., (1992): The beginning science teacher: classroom narratives of convictions and constraints. Journal of Research in Science Teaching, 29, (5), 471-485. 
BRISCOE, C., (1991): The Dynamic Interactions Among Beliefs, Role Metaphors, and Teaching Practices: A Case Study of Teacher Change. Science Education, 75, (2), 185-199.

BRISCOE, C., (1993): Using cognitive referents in making sense of teaching: A chemistry teacher's struggle to change assessment practices. Journal of Research in Science Teaching, 30, 971-987.

BRISCOE, C. y PETERS, J., (1997): Teacher collaboration across and within schools: Supporting individual change in elementary science teaching. Science Education, 81, (1), 51-65.

BRONOWSKI, J., (1987): The creative process. In Scientific genius and creativity: Readings from scientific american. (Freeman: New York). 1-8.

BRUSH, S.G., (1989): History of science and science education. Interchange, (20), 60 71.

Bunge, M., (1976): Filosofia de la Fisica. (Ariel: Barcelona).

BuNGE, M., (1980,): Epistemologia. (Ariel: Barcelona).

BURBULES, N. y LINN, M., (1991): Science education and philosophy of science: congruence or contradiction? International Journal of Science Education, 13, (3), 227241.

CACHAPUZ, A. F., (1995): Da investigaçao sobre e para professores à investigaçào com e pelos professores de ciências. En: La formación del profesorado de ciencias y matemáticas en España y Portugal. 243-254. Departamento de Didáctica de las ciencias experimentales y de las Matemáticas. Facultad de Educación. Universidad de Extremadura. Badajoz.

CAMPO, C. Y CACHAPUZ, A., (1997): Imagens de Ciência en manuais de química portugueses, Quimica Nova, 6, 23-29.

Carrascosa, J.; Fernández, I; GiL, D. y Orozco, A., (1993): Análisis de algunas visiones deformadas sobre la naturaleza de la ciencia y las características del trabajo cientifico. Enseñanza de las Ciencias. Volumen Extra. 43-46.

ChALmers, A.F., (1992): La ciencia y como se elabora. (Siglo XXI: Madrid).

Cleminson, A., (1990): Establishing an epistemological base for science teaching in the light of contemporary notions of the nature of science and of how children learn science.,Journal of Research in Science Teaching, 27, (5), 429- 445.

COBERN, W. W. y LOVING, C., (1998): The card exchange introducing the philosophy of science. In W.F. McComas (Ed): The nature of science in science education. Rationales and estrategies. (Kluwer Academic Publishers). 
DaWKings, K. R. y GlatTHORN, A. A., (1998): Using historical case estudies in biology to explore the nature of science: A professional developement program for high school teachers. In W.F. McComas (Ed). The nature of science in science education. Rationales and estrategies. (Kluwer Academic Publishers. Netherland).

Désautels, J. y LAROChelle, M., (1998a): About the epistemological posture of science teachers. In: Tiberghien A., Jossem L. y Barojas J. (Eds), Connecting Research in Physics Education with Teacher Education (ICPE Books).

DÉsAuTELs, J. y LAROCHELle, M., (1998b): The epistemology of students: The "thingified" nature of scientific knowledge.In Fraser B y Tobin K (Eds) International Handbook of Science Education, (Kluber: London)

Désautels, J., LaRochelle, M., GAGNé, B et Ruel, F., (1993): La formation a l'enseignement des sciences: le virage épistémologique. Didaskalia, 1, 49-67.

DRIVER R y OlDhaM V, (1986): A constructivist approach to curriculum development in science. Studies in Science Education, 13, 105-122.

DusCHL, R. A., (1994): Research on the history and philosophy of science. In Gabel D. (ed), Handbook of Research on Science Teaching and Learning. 445-455. (McMillan, New York).

DusCHL, R. A. y WRIGHT, E., (1989): A case study of high school teachers' decision making models for planning and teaching sicence. Journal of Research in Science Teaching. 26, 467-501.

EiChinger, D. C. y Abell, S. K. y DAGHeR, Z. R., (1997): Developing a Gradute Level Science Education Course on the Nature of Science. Science of Education, 6; 417-429.

ESTANY A., (1990): Modelos de cambio cientifico. (Editorial Crítica: Barcelona).

FERNANDEZ, I., (2000): Análisis de las concepciones docentes sobre la actividad científica: Una propuesta de transformación. Tesis Doctotal. Departament de Didàctica de les Ciencies Experimentals. Universidad de Valencia.

FERNÁNDEZ, I. y OROZCO, A., (1995): La transformación de las concepciones espontáneas sobre la ciencia. Tesis de Tercer Ciclo. Universidad de Valencia: Valencia.

FeYerabend, P., (1989): Contra el Método. (Ariel: Barcelona, 1989).

FILLON, P., (1991): Histoire des sciences et reflexion epistemologiques des elèves. Aster, $12,91-120$.

FrASER, B .J. y TOBIN, K. G., (1998): International Handbook of Science Education. (Kluwer Academic Publishers.London). 
FUrIO, C., (1994): Tendencias actuales en la formación del profesorado de ciencias. Enseñanza de las Ciencias, 12, (2), 188-199.

GabeL, D. L, (1994): Handbook of Research on Science Teaching and Learning. New York Mcmillan).

GAGLIARDI, R. Y GIORDAN, A., (1986): La historia de las ciencias: Una herramienta para la enseñanza. Enseñanza de las ciencias, Vol. 4, (3), 253-258.

Gallagher, J. J., (1991): Perspective and Practicing Secondary School Science Teachers Knowledge and Beliefs about the Philosophy of Science. Science Education, 75, (1), 121-133.

GarCIA CRUZ, C. M., (1991): La historia de la ciencia en la futura enseñanza secundaria: Reflexiones en torno al Diseńo Curricular Base. Enseñanza de las ciencias, vol. 10, (1), 115-117.

GARCIA DiAZ, J. E., (1995): Epistemología de la complejidad y enseñanza de la ecología. El concepto de ecosistema en la Ensefianza Secundaria. Tesis Doctral. Universidad de Sevilla, 1995.

GASKELL, P. J., (1992): Authentic science and school science. International Journal of Science Education, vol. 14, n. ${ }^{\circ} 3,265-272$.

GIERE R. N., (1988): Explaining Science. A cognitive approach. (The University of Chicago Press: Chicago).

GIL, D., (1983): Tres paradigmas básicos en la enseñanza de la ciencia. Enseñanza de las Ciencias, 1, (1), 26-33.

GIL, D., (1993): Contribución de la historia y la filosofla de las ciencias al desarrollo de un modelo de enseñanza/aprendizaje como investigación. Enseñanza de las Ciencias, 11 (2), 197-212.

Gil, D., (1994a): Diez años de investigación en didáctica de las ciencias: realizaciones y perspectivas. Enseñanza de las Ciencias, 12, (2), 154-164.

GIL D., (1994b): El currículo de ciencias en la educación secundaria: ¿Área o disciplinas? ¡Ni lo uno ni lo otro, sino todo lo contrario, Infancia y Aprendizaje, 65, 19-30.

GIL, D., (1996): New trends in science education. International Journal in Science Education, Vol. 18, n. ${ }^{\circ}$ 8, 889-901.

Gil, D., Carrascosa, J. Furio, C.; Martinez-Torregrosa, J., (1991): La enseñan$z a$ de las ciencias en la educación secundaria. ICE/Universidad de Barcelona. (Ed. Horsori: Barcelona).

GiL, D., Fernandez, I. y CARRASCOSA, J., (2000): La transformación de las concepciones docentes espontáneas acerca de la ciencia. En Carretero, M. (Ed). Libro pendiente de publicación. (Argentina). 
Gil D., Carrascosa, J., Dumas-carre, A., Furio, C., Gallego, N., Gene, A., Gonzalez, E., Guisasola, J., Martinez, J., Pessoa, A. Salinas, J., Tricarico, H. y VALDES, P. (1999): ¿Puede hablarse de consenso constructivista en la educación cientifica? Enseñanza de las ciencias, 17, (3), 503-512.

Gil D. Y MARTINEZ ToRregrosa J., (1999): ¿Cómo Evaluar si se hace ciencia en el aula?, Alambique, 20, 17-27.

GuILBERT, L. y MELOCHE, D., (1993): Lidée de science chez des enseignants en formation: un lieu entre l'histoire des sciences et l'hétérogénéité des visions? Didaskalia, 2, 7-30.

GIORDAN A, (1978): Observation-Experimentation: mais comment les eleves apprennent-ils? Revue Francaise de Pedagogie, 44, 66-73. Traducción española en Infancia y Aprendizaje, 1978, número 13.

GIORDAN, A. y DE VECCHI, G., (1987): Les origines du savoir. Des concepcions des apprenants aux concepts scientifiques. Neuchatel: Delachauz \& Niestlé. (Traducción castellana: Los origenes del saber. De las concepciones personales a los conceptos científicos. Sevilla: Diada, 1988).

Gould, S. J., (1987): Time's arrow time's cycle. (Harvard University Press: Cambridge. M.A.).

HAMER, D., (1985): Epistemological considerations in teaching introductory physics. Science Education, 79, (4), 313-413.

HAMMERICH, P. L., (1998): Confronting students'conceptions of the nature of science with cooperative controversy. In W.F. McComas (Ed). The nature of science in science education. Rationales and estrategies. (Kluwer Academic Publishers. Netherland).

HASHWEEH, M. Z., (1996): Effects of science teachers' epistemological beliefs in teaching. Journal of Research in Science Teaching, 33, (1), 47-63.

Hempel, C. G., (1976): Filosofia de la ciencia natural. (Alianza: Madrid).

Hewson, P. W., Kerby, H. W. y CoOK, P. A., (1995): Determining the conceptions of teaching science held by experienced high school science teachers. Journal of Research in Science Teaching, Vol. 32, N. ${ }^{\circ}$ 5, 503-520.

Hodson, D., (1985): Philosophy of science, science and science education. Studies in Science Education, 12, 25-57.

Hodson, D., (1992a): In search of a meaninful relationship: an exploration of some issues relating to integration in science and science education. International Journal of Science Education, vol. 14, 541-562. 
HoDson, D., (1992b): Assessment of practical work: some considerations in philosophy of science. Science Education, 1, (2), 115-144.

HoDsON, D., (1993): Philosophy stance of secundary school science teachers, curriculum experiences and children's understanding of science: some preliminary findings. Interchange, 24 (1\&2), 41-52.

IZQUIERDO, M., SANMARTf, N. Y ESPINET, M., (1999): Fundamentación y diseño de las prácticas escolares de ciencias experimentales. Enseñanza de las Ciencias, 17(1), 45-59.

JaCoby, B. A. y Spargo, P E., (1989): Ptolomy revisited?. The existence of a mild instrumentalism in some selected high school physical science textbooks. Interchange, (20), 33-53.

Jimenez, Aleixandre, M. P., (1995): La formación del profesorado de ciencias y matemáticas en España y Portugal. Publicación del departamento de Didáctica de las Ciencias. Universidad de Extremadura.

Kemmis, S. y Mctaggert, R., (1982): The Action Research Planner. (Deakin University Press, Geelong).

KING, B. B., (1991): Beginning Teachers Knowledge of and Artitude Towards History and Philosophy of Science. Science Education, 75, (1), 135-141.

Koballa, T. R., CraWley, F .E. y Shrigley, R. L., (1990): A summary of research in Science Education 1988. Science Education, 74, (3), 253-407.

KOULAIDIS, V. y OGBORN, J., (1995): Science teacher philosophical assumptions: how well do we understand them? International Journal Science Education, Vol 17, N. ${ }^{\circ}$ 3, 273-283.

KUHN, TH. S., (1971): La estructura de las revoluciones cientificas. (Fondo de cultura económica: México).

LAKATOS, I., (1982): Historia de la ciencia y sus reconstrucciones racionales. (Tecnos: Madrid).

LAKATOS, I., (1989): La metodología de los programas de investigación cientifica. (Alianza Editorial: Madrid).

LAKIN, S. y Wellington, J., (1994): Who will teach the "nature of sciencen?. Teachers view of science and their implications for science education. International Journal of Science Education, 16, (2), 175-190.

LAUDAN, L., (1984): Science and values: tha aims of science and their role in the scientific debate University of California Press: Berkeley).

LEDERMAN, N. G., (1992): Students' and teachers' conceptions of the nature of science: A review of the research. Journal of Research in Science Teaching, 29, pp.331359. 
LeDERMAN, N. G y ABD-EL-KHALICK, F., (1998): Avoiding de-natured science: Activities that promote undrestandings of the nature of science. In W.F. In W.F. McComas (Ed). The nature of science in science education. Rationales and estrategies. (Kluwer Academic Publishers. Netherland).

LEMBerger, J., Hewson, p. Y PARK, H., (1999): Relationships between prospective secondary teachers' classroom practice and their conceptions of Biology and teaching science. Science Education, 83(3), 347-371.

LINDER, C. J., (1992): Is teacher-reflected epistemology a source of conceptual difficulty in phisics?. International Journal of Science Education, Vol. 14, N. $.^{\circ} 1,111$ 121.

Loving, C. C., (1991): The Scientific Theory Profile: A Philosophy of Science models for Science Teacher. Journal of Research in Science Teaching, 28, (9), 823-838.

LYoNS, L. L.; FreitAG, P. K. y Hewson, P. W., (1997): Dichotomy in thinking. Dilema in actions: Researcher and teacher perspectives on a chemitry teaching practice. Journal of Research in Science Teaching, 34, (3), 239-254.

MARTIN, B., KASS, H. y BROUWER, W., (1990): Authentic Science: A diversity of meanings. Science Education, 74, (5), 541-554.

MATSON, J. O. y PARSON, S., (1998): The nature of science: achieving scientific literacy by doing science. In W.F. McComas (Ed). The nature of science in science education. Rationales and estrategies. (Kluwer Academic Publishers. Netherland).

MATTHEWs, M. R., (1991): Un lugar para la historia y la filosofía en la enseñanza de las ciencias. Comunicación, Lenguaje y Educación, 11-12, 141-155.

MatTHEws, M. R., (1994): Historia, filosofía y enseñanza de las ciencias: La aproximación actual. Enseñanza de las Ciencias, 12, (2), 255-277.

MATTHEws, M. R., (1997): Editorial. Science and Education, (6), 323-329.

MATTHEWS, M. R., (1998): Foreword and introduction. In W.F. McComas (Ed). Science and Technology Education Library. (1998): "The Nature of Science in Science Education: Rationales and Strategiesm. Introduction. (Kluwer Academic Publishers. Netherland).

McCOMAS, W. F., (1998): The nature of science in science education. Rationales and In W.F. McComas (Ed). The nature of science in science education. Rationales and estrategies. (Kluwer Academic Publishers. Netherland).

McCOMAS, W. F., (1998a): The principal elements of the nature of science: Dispelling the myths. In W.F. McComas (Ed). The nature of science in science education. Rationales and estrategies. (Kluwer Academic Publishers. Netherland). 
McCOMAS, W. F., (1998b): A thematic introduction to the nature of science: The rationale and content of a course for science educators. In W.F. McComas (Ed). The nature of science in science education. Rationales and estrategies. (Kluwer Academic Publishers. Netherland).

McCOMAS, W. F., ClOUGH, M. P. y AlmazroA, H., (1998): The role and character of the nature of science in science education. In W.F. McComas (Ed). The nature of science in science education. Rationales and estrategies. (Kluwer Academic Publishers. Netherland).

McCOMAS, W. F. y OLSON, J., (1998): The nature of science in international science education standarsdocuments. In W.F. McComas (Ed). The nature of science in science education. Rationales and estrategies. (Kluwer Academic Publishers. Netherland).

MEICHTRY, Y., (1998): Elementary science teaching methods: Developing and measuring student view about the nature of science. In W.F. McComas (Ed). The nature of science in science education. Rationales and estrategies. (Kluwer Academic Publishers. Netherland).

Mellado, V., (1996): Concepciones y prácticas de aula de profesores de ciencias en formación inicial de primaria y secundaria. Enseñanza de las Ciencias, 14, (3), 289302.

MEllado, V., (1997): Preservice teachers' classroom practice and their conceptions of the nature of science. Science Education, 6, 331-354.

MELLADO, (1998): The classroom practice of preservice teachers and their conceptions of teaching and learning science. Science Teacher Education, 82, 197-214.

Mosterín J., (1990): Prólogo al libro de Estany A., Modelos de cambio cientifico. (Editorial Crítica: Barcelona).

NADEAU, R. y DÉSAUTELS, J., (1984): Epistemology and the teaching of science. (Science Council of Canada. Ottawa).

NewTON, D. P. y NEwTON, L. D., (1992): Young children's perceptions of science and scientist. International Journal in Science Education, vol. 14, n. 3,331 348.

NOTT, M. y WELLINGTON, J., (1998): A programe for developing understanding of the nature of science in teacher education. In W.F. McComas (Ed). The nature of science in science education. Rationales and estrategies. (Kluwer Academic Publishers. Netherland).

NussBaum, J., (1989): Classroom conceptual change: philosophical perspectives. International Journal in Science Education, vol. 11, Special Issue, 530-540. 
OrOZCO, A. y FERNANDEZ, I., (1995): El problema de las concepciones espontáneas sobre la ciencia. Tesis de Tercer Ciclo. Universidad de Valencia: Valencia.

OTERO, J. (1985): Assimilation problems in traditional representation of scientific knowledge. European Journal of Science Education, 7, (4), 361-369.

PAIXÃO, M. F. y CACHAPUZ, A., (1998): Dimensión epistemológica de los programas de Física y Química e implicaciones en las prácticas de enseñanza: ¿Qué lectura hacen los profesores?. En Banet, E. Y De Pro, A. (Eds), Investigación e Innovación en la Enseñanza de las Ciencias, Vol. 1, 284- 293.

PAIXÃO, M. F. y CACHAPUZ, A., (1999): Challenges on science teacher education for the new century: an approach based on the epistemology of curricular themes. Comunicación presentada en la $24^{\text {th }}$ ATEE Anual Conference. Leipzig.

PEDRINACI, E., (1994): Epistemologia, historia de las ciencias y abejas. Investigación en la escuela, 23, 95-102.

PENICK, J. E. y YAGER, R. E., (1986): Trends in science education: Some observations of exemplary programmes in the U.S.A. European Journal of Science Education, 8, 1-8.

Perales, F J. y CAN̂AL, P., (2000): Didáctica de las ciencias experimentales. Teoria y práctica de la enseñanza de las ciencias. (Marfil: Alcoy).

PIAGET, J., (1970): La epistemología genética. (Redondo: Barcelona).

POPPER, K. R., (1962): La lógica de la investigación cientifica (Tecnos: Madrid).

POMEROY, D., (1993): Implications of teachers' beliefs about the nature of science: Comparison of the beliefs of scientits, secondary science teachers, and elementary teachers. Science Education, 77, (3), 261-278.

PORLAN, R., (1989): Teoría del conocimiento, teoría de la enseñanza y desarrollo profesional. Las concepciones epistemológicas de los profesores. Tesis Doctoral. Universidad de Sevilla.

PORLAN, R. y RIVERo, A., (1998): El conocimiento de los profesores. (Diada Editora. Sevilla, 1998).

ROTH, W. M. y LUCAS, K. B., (1997): From "Truth" to "Invented Reality": A Discourse Analysis of High School Physics Students' Talk about Scientific Knowledge. Journal of Research in Science Teaching, Vol. 34, n. ${ }^{\circ} 2,145-179$.

ROTH, W. M. y RoYCHONDHURY, A., (1994): Students' Epistemologies and Views about Knowing and Learning. Journal of Research in Science Teaching Vol. 31, n. $1,5-30$.

RUBA, P. A. y HARKNERSS, W. L., (1993): Examination of preservice and in-service secondary science teachers'beliefs about Science/Tecnology/Society interactions. Science Education, 77, (4), 407-431. 
RUgGieri, R. TARSITANI, C. Y VICENTINI, M., (1993): The images of science of teachers in latin countries. International Journal of Science Education, vol. $15, \mathrm{n}^{\circ} 4$, 383-393.

SANMARTI N., MAUR T., IzQUierdo M. E GOMEZ I., (1990): Los procedimientos, Cuadernos de Pedagogia, 180, 28-32.

Selley, N. J., (1989): The philosophy of school science. Interchange, 20, (2), 24-32.

Solomon, J., (1991): Teaching about the nature of science in the British National Curriculum. Science Education, 75, (1), 95-103.

SOLOMON, J., DUVEen, J. y SCOTT, L., (1994): Pupils' images of scientific epistemology. International Journal of Science Education, Vol. 16, N. ${ }^{\circ} 3,361-373$.

SONGER, N. B. y LINN, M. C., (1991): How do students views of science influence knowledge integration? Journal of research in Science Teaching, 28, (9), 761-784.

SPECTOR, B., STRONG, P. y LA PORTA, T., (1998): Teaching the nature of science as an element of science, tecnology and society. In W.F. McComas (Ed). The nature of science in science education. Rationales and estrategies. (Kluwer Academic Publishers. Netherland).

STINNER, A., (1992): Science textbooks and science teaching: from logic to evidence. Science Education, 76, (1), 1-16.

SUTTON, C., (1998): New peerspectives on language in science. In: B.J. Fraser and K.G. Tobin (eds). International Handbook of Science Education. (Kluwer Academic Publishers. Great Britain).

Thomaz, M. F.; Cruz, M. N.; Martins, I. P. y Cachapuz, A. F., (1996): Concepciones de futuros profesores del primer ciclo de primaria sobre la naturaleza de la ciencia: Contribuciones de la formación inicial. Enseñanza de las Ciencias, 14, (3), pp.315-322.

Tobin, K. G. y Mcrobrie, C. J., (1997): Beliefs about the nature of science and the enacted science curriculum. Science and Education, (6), 355-371.

Tobin, K. G., TipPINS, D. J., y Gallard, A. J., (1994): Research on Instruccional Strategies for Teaching Science. In D.L. Gabel (Ed) Handbook of Research on Science Teaching and Learning. New York: National Science Teachers Association.

ToBIN, K. G., TIPPINS, D. J., y HoOK, K., (1994): Referents for changing a science curriculum: A case study of one teacher's cange in beliefs. Science Education, 3, pp 245-264.

Toulmin, S., (1977): La comprensión humana. I: el uso colectivo y la evolución de los conceptos (Alianza: Madrid). 
Traver, M J., (1996): La història de les ciències en l'ensenyament de la Física i la Química. Tesis Doctoral. Departament de Didàctica de les Ciencies Experimentals. Univeridad de València, 1996.

YAGER, R. E. y PENICK, J. E., (1983): Analysis of the current problems with school science in the U.S.A. European Journal of Science Education, 5, 459-463. 